\title{
Minimizing the number of remotely controlled switches when the planning the reconfiguration of a primary distribution network
}

\author{
Irina Golub ${ }^{1, *}$, Oleg Voitov ${ }^{1}$, Evgeny Boloev ${ }^{1}$ and Lyudmila Semenova ${ }^{1}$ \\ ${ }^{1}$ Energy Systems Institute of Siberian Branch of the Russian Academy of Sciences, Department of Electric Power System , 664033 \\ Lermontov str., 130, Irkutsk, Russia
}

\begin{abstract}
The work shows the possibility to select the permanent configuration of a primary distribution network at which power losses during a week with maximum load and during a week with minimum load the power losses would be close to losses at optimum hourly reconfiguration. Data on the mix of loop branches obtained during the reconfiguration algorithm operation is used for selecting the tie-switches that allow both the regime restoration after emergency opening of a sectionalized switch, and acceleration of this process, especially if the tie-switch is remotely-controlled. The IEEE 33-node test systems are used to demonstrate the proposed algorithms.
\end{abstract}

\section{Introduction}

Reconfiguration of a primary distribution network is the most recognized method of optimizing its topology. The main purpose of reconfiguration is reliable power supply of consumers, which in case of emergency opening of a normally closed sectionalizing switch in the primary distribution network is performed by closing a normally opened tie-switch to ensure operation of a weakly closed primary distribution network as an opened one. Other criteria of optimum reconfiguration may include minimization of power losses and voltage losses, minimization of the network maintenance costs and increase of its transfer capacity.

Reconfiguration as a method for power loss reduction in the city distribution network was first proposed in [1]. Since then a large number of reconfiguration algorithms has been developed that, according to [2], can be divided into four groups according to combination of heuristic and optimization methods, heuristic or meta heuristic methods only, and mathematical algorithms.

In the previous studies [2] on reconfiguration for power loss reduction all the switches were deemed to be remotely controlled, which does not correspond to reality as switches used in conventional primary distribution networks are switched over manually for reconfiguration.

It does not allow frequent switchings and configuration of a large number of distribution networks remains constant during the season. Switchings for losses minimization in this case can be implemented, for example, for the regime of maximum or minimum loads.

With transition to active distribution networks the manual switches shall be replaced by automatic or remotely controlled ones, which would allow more frequent reconfiguration in the hourly, weekly, daily, monthly or seasonal time intervals. Remotely controlled switches are important for power supply restoration after a permanent fault [3]. Occurrence of transient processes and over voltage during switching is a negative factor limiting the frequency of reconfigurations and having a negative impact on the network reliability [4].

The main obstacle that does not allow the use of only remotely controlled switches for reconfiguration is high cost, a limited number of allowable switchings and, hence, high cost of an individual switching. According to [5], cost of a remotely controlled switch is as high as $\$ 4,000$. Its service life being 40 years, the maximum number of switchings is 10,000 [6], i.e., 250 switchings a year, or 5 switchings a week. Unjustifiably high costs of replacing all the switches by switches controlled from a dispatching center by a distribution network operator make the problem of determining the minimum number of switches used for the network reconfiguration important [7].

According to [8], for hourly reconfiguration of a distribution network that ensures minimum daily power losses, availability of renewable power generation has a greater impact on power losses reduction than reconfiguration, but with introduction of renewable power generation the number of switchings during reconfiguration increases considerably. It also shows the possibility of selecting the configuration of a primary distribution network at which power losses during the day would be close to daily losses at optimum hourly reconfiguration. In the present paper this idea extends to selection of the permanent configuration at which during a week with maximum load and during a week with minimum load the power losses would be close to losses at optimum hourly reconfiguration.

A reconfiguration algorithm offered by the authors [9] is based on the methods of determining of a maximum spanning tree [10] on the network graph and a mix of branches of independent loops by their chords

*Corresponding author: golub@isem.irk.ru 
[11] with the corresponding normally opened tie switches. A reconfiguration algorithm is included into a fast-acting program for loadflow calculation SDO-7 [12].

Data on the mix of loop branches obtained during the reconfiguration algorithm operation is used for selecting the tie-switches that allow both the regime restoration after emergency opening of a sectionalized switch, and acceleration of this process, especially if the tie-switch is remotely-controlled.

The article is structured in the following way. Section 1 is devoted to study of the impact of optimum hourly reconfiguration on power losses in the test distribution network with and without renewable generation sources. In Section 2 these losses, as reference ones, are compared to losses at selection of a permanent configuration of a distribution network. Section 3 solves the problem of regime recovery after permanent fault at any sectionalizing switch at a permanent configuration of a distribution network. The effort is made to solve the problem of assessing the feasibility and acceptability of the post-emergency regime.

\section{Power losses during reconfiguration}

To demonstrate the options of a permanent configuration of a distribution network and to analyze factors affecting power losses in it, a 33-node circuit is used Fig. 1 [13] that includes 32 sectionalizing and 5 tie-switches. Data on the rated loads of this diagram were taken from [13].

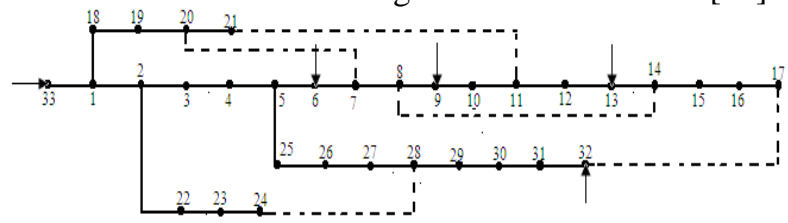

Fig. 1. A diagram of a 33-node distribution network; dashed lines are branches with tie-switches

Fig. 2 shows profiles of hourly curves with maximum Fig. $2(a)$ and minimum Fig. $2(b)$ weekly loads that are built using standard graphs of daily change in the industrial, commercial and municipal loads that were proposed in [2]. Daily graphs of hourly change in the active generating power of renewable generation sources in Nodes 6, 9,13, and 32 Fig. 3 that were taken from [2] were deemed to be constant for every weekday.

Fig. 4 presents curves of losses in the distribution network under the lack or availability of renewable generation and reconfiguration in it within weeks with regimes of maximum and minimum loads that confirm notable impact of renewable generation on the reduction of power losses.
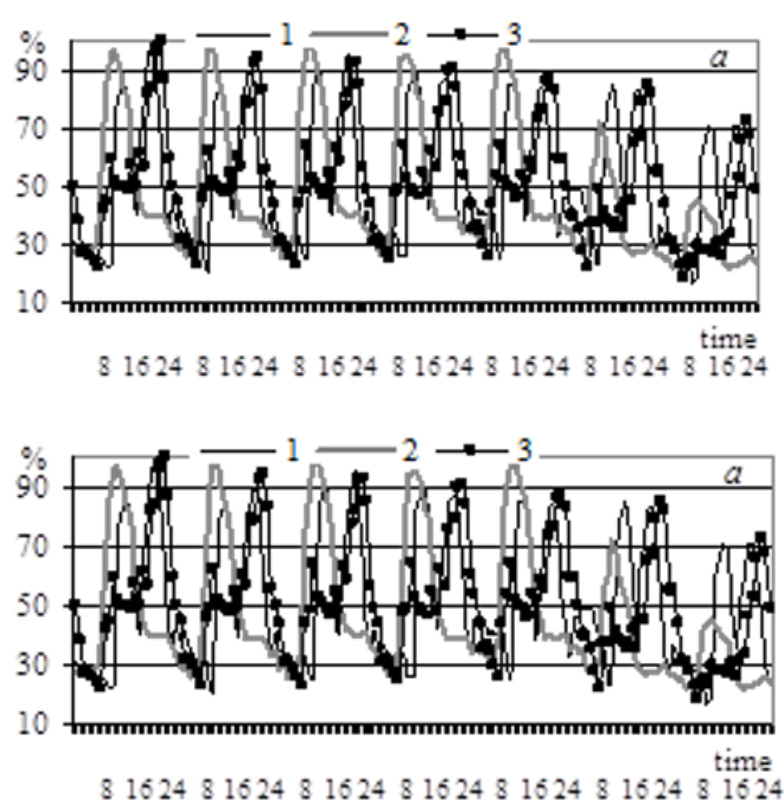

Fig. 2. Curves for maximum $(a)$ and minimum $(b)$ weekly industrial (1), commercial (2), and municipal (3) loads

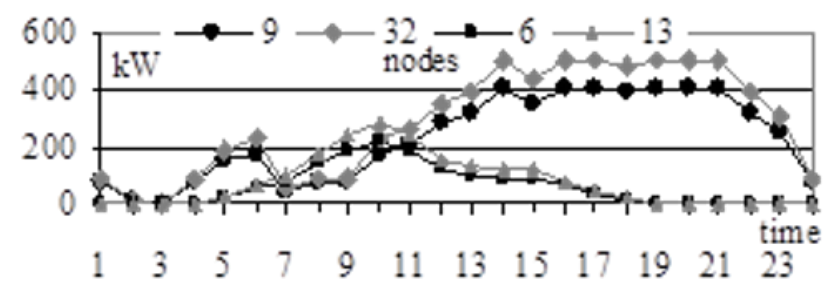

Fig. 3. Change in the active power of renewable generation sources in the distribution network nodes within a day
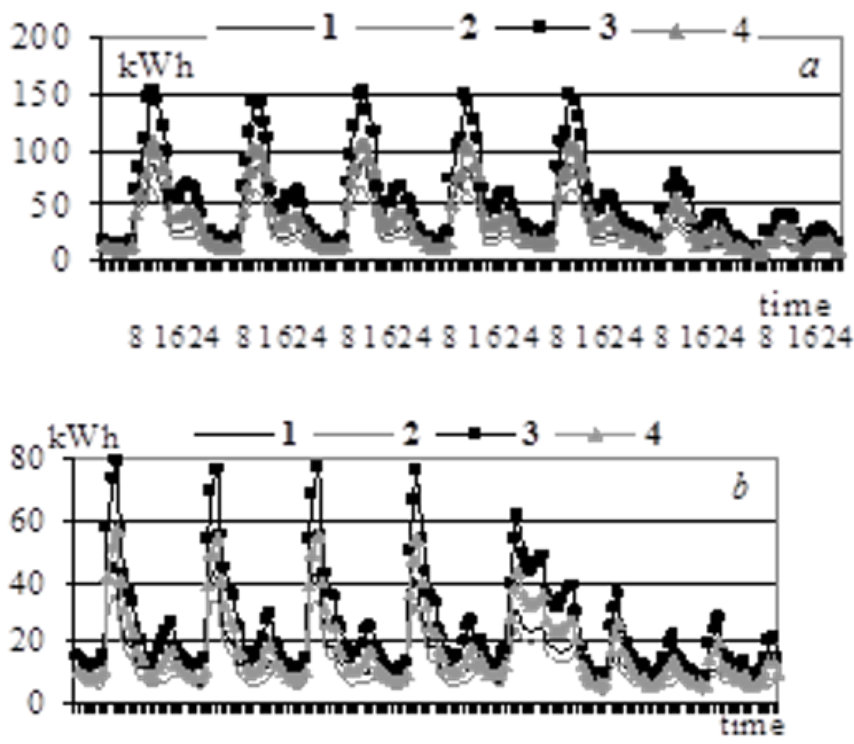

81624816248162481624816248162481624

Fig. 4. Change in the power losses in the regime of maximum (a) and minimum (b) loads in the primary distribution network within a week: With renewable generation sources under the lack (1) and availability (2) of reconfiguration; without renewable generation sources under the lack (3) and availability (4) of reconfiguration 


\section{Selection of permanent configuration of a distribution network with minimum losses}

3.1 An option with availability of renewable generation sources in the distribution network with maximum loads.

The number of switchings within a week in the studied network with renewable generation at optimum hourly reconfigurations equals 530, or 76 switchings a day, on the average. Compare: the number of switchings in the network without renewable generation for the same period is 132. Let us study the chances of selecting such permanent configuration of a distribution network that would ensure loses close to those at optimum hourly reconfiguration during a week.

To determine the permanent configuration for each day of the week with maximum loads, the following calculations have been performed. Configuration ensuring the minimum power losses was determined using the given hourly loads and generations Fig. 4. Then daily power losses were calculated for optimum configurations of each hour subject to their invariability within the day.

Fig. 5 demonstrates daily losses for seven days of the week starting from Monday, at permanent configuration of a distribution network that coincides with every hourly configuration obtained for it.

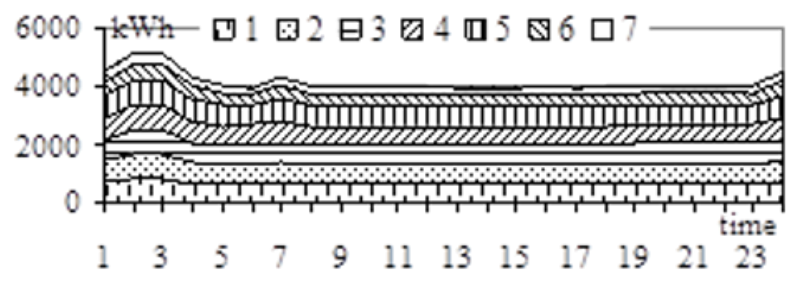

Fig. 5. Values of daily looses for each day of the week at permanent configuration of a distribution network that coincides with optimum hourly configuration obtained for it

For example, minimum losses for the first day of the week correspond to the 15th hour, and network configuration is defined by tie-switches (1: 5-6, 9-10, 1314, 17-32, 24-28). Configuration with minimum losses of the second day with tie-switches (2: 6-7, 7-8, 13-14, 17-32, 27-28) coincides with configurations of the 4th, 6 th and 7 th days. Configuration with minimum losses of the third day is determined by tie-switches (3: 6-7, 9-10, 13-14, 24-28, 29-30), and configuration of the 5th day is determined by tie-switches (4: 5-6, 10-11, 13-14, 17-32, 24-28).

Fig. 6 shows changes in the power losses within a week for each out of four selected configurations; Fig. 7 presents aggregate values of weekly losses compared to losses under the lack of reconfiguration and losses at optimum daily reconfiguration.

Comparison of losses Fig. 7 distinguishes configuration of the first day of the week. Subject to its permanency the losses within a week are $257 \mathrm{kWh}$ higher than losses at optimum hourly reconfiguration requiring 530 switchings.

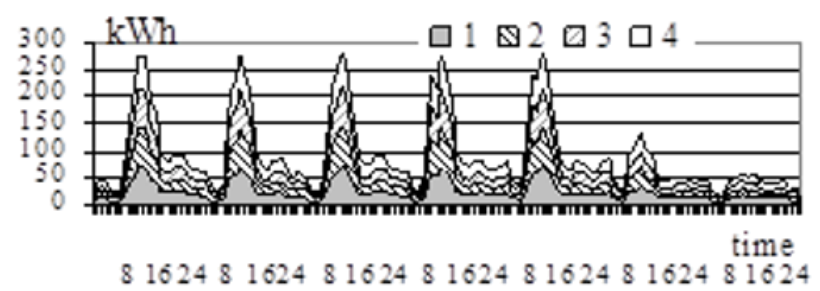

Fig. 6. Change in the power losses within a week for each out of four selected configurations

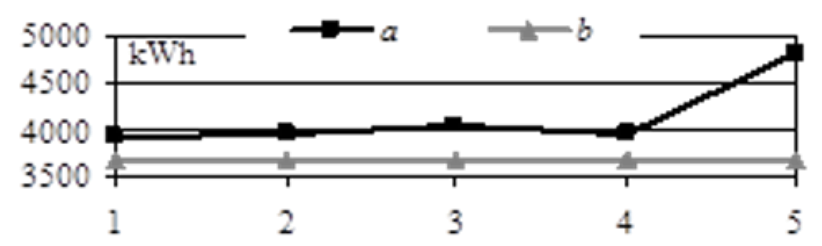

Fig. 7. Comparison of aggregate weekly power losses in the distribution network: $a$ - for four options with permanent configuration; 1-4 and 5-under the lack of reconfiguration; $b$ - at optimum hourly reconfiguration

3.2 An option without renewable generation sources in the distribution network with maximum loads.

For the option of a distribution network without renewable generation it was found out that on week days it corresponds to configuration with tie-switches (1: 6-7, $8-9,13-14,24-28,31-32)$, and on weekends the configuration (2: 6-7, 8-9, 13-14, 27-28, 31-32) differed from weekdays configuration by one switch.

Comparison of losses at permanent configurations with losses at optimum weekly configuration Fig. 8 has shown that losses at the first configuration exceed optimum ones by $64 \mathrm{kWh}$, at second one - by $82 \mathrm{kWh}$, whereas losses in the network without reconfiguration exceed losses at optimum reconfiguration by $2646 \mathrm{kWh}$.

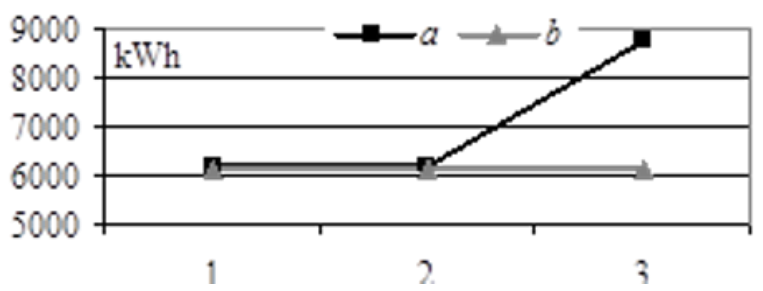

Fig. 8. Comparison of aggregate weekly power losses in the distribution network: $a$ - for two options with permanent configuration; 1-2 and 3-under the lack of reconfiguration; $b$ - at optimum hourly reconfiguration

3.3 An option with the availability of renewable generation sources in the distribution network with minimum loads.

For the regime of minimum load at optimum hourly reconfiguration of the network within a week the reduction of losses as against losses under the lack of reconfiguration accounted for $612 \mathrm{kWh}$ at 572 switchings. 
Three configurations with minimum daily losses were singled out for the considered option. The first configuration is common for the first, second, sixth and seventh days and is determined by tie-switches (1: 6-7, $7-20,8-14, \quad 17-32,27-28)$. The second configuration with switches (2: 6-7, 7-8, 12-13, 17-32, 27-28) corresponds to the third and fourth days. The third configuration corresponds to the fifth day and is determined by tie-switches (3: 5-6, 7-8, 13-14, 17-32, 27-28).

Comparison of aggregate weekly power losses for three options with permanent configuration allowed identification of the second configuration whose losses differ from losses at optimum reconfiguration by 170 kWh Fig. 9.

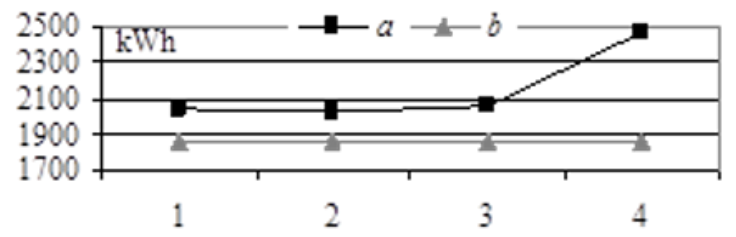

Fig. 9. Comparison of aggregate weekly power losses in the distribution network: $a$ - for three options with permanent configuration; 1-3 and 4-under the lack of reconfiguration; $b$ - at optimum hourly reconfiguration

\subsection{An option without renewable generation sources in} the distribution network with minimum loads.

Calculations were made for the regime of minimum load without renewable generation Fig. 10.

Analysis have shown that the first out of two selected configurations corresponding to weekdays include tieswitches (6-7, 8-9, 13-14,24-28,31-32), and the second configuration for weekends differs from it by one tieswitch 27-28 only that shall replace tie-switch 24-28.
Thus, configurations with minimum weekly power losses for regimes with maximum and minimum loads coincided.

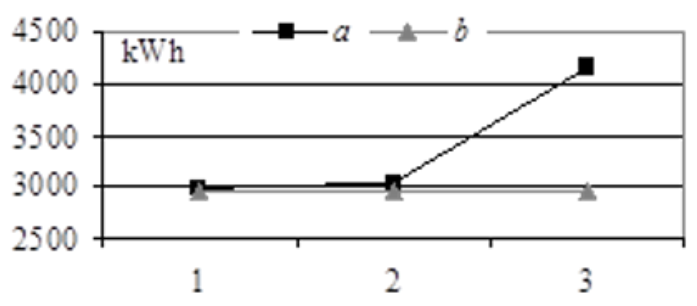

Fig. 10. Comparison of aggregate weekly power losses in the distribution network: $a$ - for two options with permanent configuration; 1-2 and 3-under the lack of reconfiguration; $b$ - at optimum hourly reconfiguration

Comparison of aggregate weekly power losses have shown that losses at the first configuration differ from losses in the network with optimum reconfiguration by $27 \mathrm{kWh}$, at the second configuration - by $63 \mathrm{kWh}$, whereas losses in the network without reconfiguration exceed losses in the network with reconfiguration by $1197 \mathrm{kWh}$.

\subsection{Selection of permanent configuration of a distribution network with minimum losses}

Results of selection of a permanent configuration of a distribution network with renewable generation for the regime with maximum and minimum loads are given in Table 1 that also includes results of additional study of weekly losses in the regime of maximum loads for three configurations of the regime of minimum loads, and in the regime of minimum loads for four configurations of the regime of minimum loads.

Table 1. Power losses during the week with maximum loads and the week with minimum loads, aggregate power losses for a two-

\begin{tabular}{|c|c|c|c|c|}
\hline \multirow{2}{*}{$№$} & Mix of tie-switches & Power losses & Power losses & \multirow{2}{*}{$\begin{array}{c}\text { Aggregate } \\
\text { power losses }\end{array}$} \\
\cline { 2 - 5 } & For regime with maximum load & Maximum load & Minimum load & 6055.471 \\
\hline 1 & $1: 5-6,9-10,13-14,17-32,24-28$ & $\mathbf{3 9 3 5 . 4 5 4}$ & 2120.017 & $\mathbf{5 9 7 7 . 1 4}$ \\
\hline 2 & $2: 6-7,7-8,13-14,17-32,27-28$ & 3948.498 & $\mathbf{2 0 2 8 . 6 4 2}$ & 6741.425 \\
\hline 3 & $3: 6-7,9-10,13-14,24-28,29-30$ & 4056.291 & 2685.134 & 6069.197 \\
\hline 4 & $4: 5-6,10-11,13-14,17-32,24-28$ & 3946.542 & 2122.655 & Minimum load \\
\hline 5 & For regime with minimum load & Maximum load & 2038.154 & 6115.705 \\
\hline 6 & $1: 6-7,7-20,8-14,17-32,27-28$ & 4077.551 & $\mathbf{2 0 3 2 . 7 4 5}$ & 6006.528 \\
\hline 7 & $2: 6-7,7-8,12-13,17-32,27-28$ & 3973.783 & 2056.139 & $\mathbf{5 9 7 4 . 0 3 3}$ \\
\hline
\end{tabular}

Comparison of losses for two weeks with maximum and minimum loads shows that minimum losses correspond to the third configuration of the regime with minimum loads. Minimum weekly power losses for regimes with minimum and maximum loads that equal $6182.563 \mathrm{kWh}$ and $2120.201 \mathrm{kWh}$, respectively, in the network without renewable generation sources are observed at the same permanent configuration with tieswitches 6-7, 8-9, 13-14, 24-28, 31-32. 


\section{Selection of tie-switches for the regime restoration at emergency opening of sectionalized switches network}

For final selection of permanent configuration of a distribution network, make sure that at emergency opening of any sectionalized switch a short circuit of the corresponding tie-switch would lead neither to occurrence of unacceptable voltage deviations in the distribution network, nor to rated temperature-rise currents. In case of limitations violation the controls that ensure permissible post-emergency regime shall be selected.

A reconfiguration algorithm used in this work is based on building the spanning tree on the network graph and on determination of the mix of branches of independent loops by their chords, and ideally suits determination of tie-switches used for power supply recovery in case of emergency opening of a sectionalized switch.

An algorithm for selection of tie switches options includes the following steps:

1. Branches with the given tie switches specify the mix of the network graph chords, the remaining branches being the branches of a spanning tree.

2. For determining the branches of each independent loop by its chord, a sub-matrix $N_{t}$ of a block of trees of the second matrix of incidences shall be built [10],

$$
N_{t}=-M_{c h}^{\mathrm{T}}\left(M_{t}^{\mathrm{T}}\right)^{-1}
$$

the number of rows in which would be equal to the number of chords, and the number of columns would be equal to the number of branches in the spanning tree, where $M_{t}$ and $M_{c h}$ are sub-matrices of the first incidence matrix $M=\left(M_{t} M_{c h}\right)$ that correspond to the tree branches and chords. Formula (1) forms the base for a topology algorithm [11] for building both the submatrix $\left(M_{t}^{\mathrm{T}}\right)^{-1}$, and the second incidence matrix $N=\left(N_{t} N_{c h}\right)$.

The same branch of the spanning tree can be within several loops; in this case after the emergency opening of a sectionalized switch the regime may be recovered by closing any related general loop of tie-switches. Minimum hourly power losses and minimum voltage deviations may be the criteria for selecting the best option.

Let us analyze the process of network reconfiguration with renewable generation sources whose configuration corresponds to the first mix of tie-switches for the regime of maximum loads Fig. 11 at emergency opening of any sectionalized switch. The studied configuration corresponds to optimum configuration of the 15-th hour of the first day of the week with maximum loads.

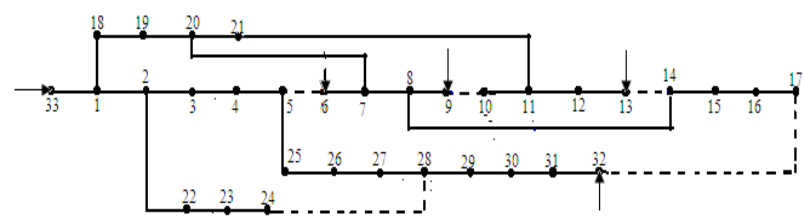

Fig. 11. Configuration of a distribution network corresponding to the first mix of tie-switches for the regime of maximum loads

Table 2. Options of tie switches that can be used for regime recovery at emergency opening of sectionalized switches in the distribution network with renewable generation sources

\begin{tabular}{|c|c|c|c|c|c|c|c|}
\hline \multirow{2}{*}{$\begin{array}{l}\text { Sectionalized } \\
\text { switches }\end{array}$} & \multicolumn{4}{|c|}{ Options of tie switches } & \multirow{2}{*}{$\begin{array}{l}\text { Sectionalized } \\
\text { switches }\end{array}$} & \multicolumn{2}{|c|}{ Options of tie switches } \\
\hline & 1 & 2 & 3 & 4 & & 1 & 2 \\
\hline $1-2$ & $5-6$ & $17-32$ & & & $14-15$ & $17-32$ & \\
\hline $1-18$ & $5-6$ & $17-32$ & & & $15-16$ & $17-32$ & \\
\hline $2-3$ & $5-6$ & $17-32$ & $24-28$ & & $16-17$ & $17-32$ & \\
\hline $2-22$ & $24-28$ & & & & 18-19 & $5-6$ & $17-32$ \\
\hline $3-4$ & $5-6$ & $17-32$ & 24-28 & & $19-20$ & $5-6$ & $17-32$ \\
\hline $4-5$ & $5-6$ & $17-32$ & $24-28$ & & $20-21$ & $9-10$ & $13-14$ \\
\hline $5-25$ & $17-32$ & $24-28$ & & & $22-23$ & $24-28$ & \\
\hline $6-7$ & $5-6$ & & & & $23-24$ & $24-28$ & \\
\hline $7-8$ & $9-10$ & 13-14 & $17-32$ & & $25-26$ & $17-32$ & $24-28$ \\
\hline $7-20$ & $5-6$ & $9-10$ & 13-14 & $17-32$ & $26-27$ & $17-32$ & $24-28$ \\
\hline $8-9$ & $9-10$ & & & & $27-28$ & $17-32$ & $24-28$ \\
\hline $8-14$ & 13-14 & $17-32$ & & & $28-29$ & $17-32$ & \\
\hline $10-11$ & $9-10$ & & & & $29-30$ & $17-32$ & \\
\hline $11-12$ & $13-14$ & & & & $30-31$ & $17-32$ & \\
\hline $11-21$ & $9-10$ & $13-14$ & & & $31-32$ & $17-32$ & \\
\hline $12-13$ & $13-14$ & & & & & & \\
\hline
\end{tabular}

Possible switchings are given in Table 2. For example, at emergency opening of a sectionalized switch 7-20 within four independent loops: 1: 5-6, 5-4, 4-3, 3-2, 2-1, 1-18, 18-19, 19-20, 20-7, 7-6; 2: 9-10: 9-8, 8-7, 720, 20-21, 21-11, 11-10; 3: 13-14,:13-12, 12-11, 11-21, 21-20, 20-7, 7-8, 8-14; 4: 17-32: 32-31, 31-30, 30-29, 29-28, 28-27, 27-26, 25-5, 5-4, 4-3, 3-2, 2-1, 1-18, 18$19,19-20,20-7,7-8,8-14,14-15,15-16,16-17$; power supply can be restored by closing the tie-switch corresponding to the chord (in bold) of any out of four independent loops. Should tie-switches be considered as candidates for substitution by remotely-controlled ones, then from Table 2 it follows that four remotelycontrolled switches instead of five ones are sufficient for power supply restoration. Actually, in the emergency situations when switch $24-28$ is offered as an alternative 
for power supply restoration, tie-switch 17-32 can be used instead of it.

Analysis of voltages and currents in the week with maximum loads has shown that the emergency opening of a sectionalizing switch 1-2 constitutes the heaviest case as after power supply recovery by closing the tieswitch 5-6, the voltage drops occurred that exceeded permissible ones

$$
\left(U_{\text {rated }}-U\right) \cdot 100 \% / U_{\text {rated }} \leq 10 \% \text {. }
$$
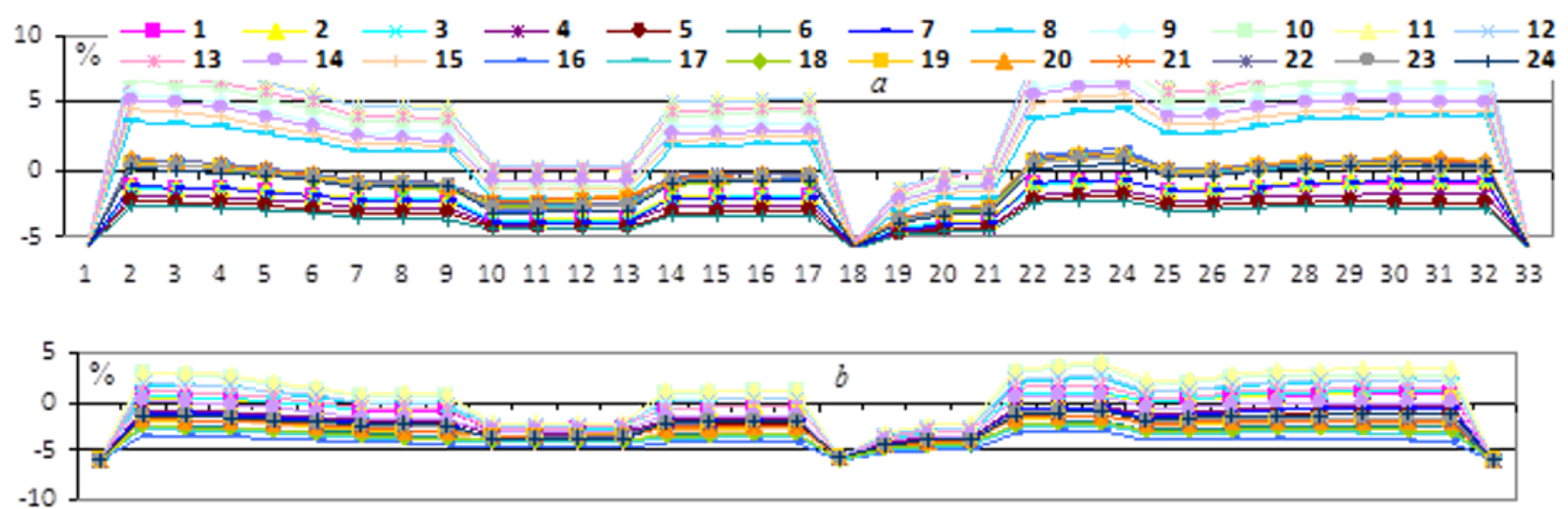

$\begin{array}{llllllllllllllllllllllllllllllllll}1 & 2 & 3 & 4 & 5 & 6 & 7 & 8 & 9 & 10 & 11 & 12 & 13 & 14 & 15 & 16 & 17 & 18 & 19 & 20 & 21 & 22 & 23 & 24 & 25 & 26 & 27 & 28 & 29 & 30 & 31 & 32 & 33\end{array}$

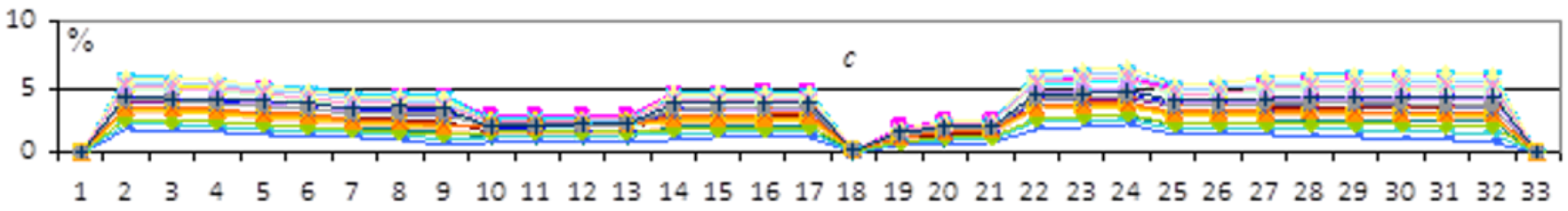

Fig. 12. Deviation of voltages in the nodes of the distribution network Fig. 11 after opening the tie 1-2 and closing the tie 5-6 under $6 \%$ voltage increase in the power supply node for the first $(a)$ and sixth $(b)$ days of the week at rated voltage in the power supply node for the seventh day of the week $(c)$

As a possible control to ensure this requirement was chosen voltage increase in power supply node 33 by $6 \%$ in relation to the rated voltage, Fig. 12.

\section{Summary}

The proposed approach to selection of permanent configuration of a distribution network for reducing the power losses in it, and power supply restoration in case of emergencies is one of possible solutions that can be implemented at the intimal stage of transition from a traditional passive to an active distribution network. Comparison of such economic indicators as costs and power losses, switch costs, consumers' profit reduction due to power supply interruption, and assessment of the network reliability before and after reconfiguration shall be the most important criteria of the reconfiguration efficiency. Algorithms of optimum reconfiguration and determination of switchings for power supply restoration in case of emergency have low labor intensity and can be introduced into programs for computing the power flows in the distribution network.

The work is done in the framework of the project III.17.4.2. program of fundamental research SB RAS, registration number AAAA-A17-117030310438-1.

\section{References}

1. A. Merlin, H. Back Search, PSCC 5, 1 (1975)

2. M.R. Dorostkar-Ghamsari, M. Fotuhi-Firuzabad, M. Lehtonen, A. Safdarian, IEEE Trans. on Power Systems 31, 1879 (2016)

3. S. Lei, J. Wang, Y. IEEE Trans. on Power Systems 33, 3129 (2018)

4. V. Spitsa, X. Ran, R. Salcedo J.F. Martinez, R.E. Uosef, F. León, D. Czarkowski, Z. Zabar, IEEE Trans. Smart Grid 3, 887 (2012)

5. S. A. Yin, C. N. IEEE Trans. Power Syst. 24, 652 (2009)

6. Switchgear Solutions, Schneider Electric Inc.

7. S. Lei, Y. Hou, F. Qiu, J.Yan, IEEE Trans. on Sustainable Energy 9, 420 (2018)

8. I.I. Golub, O.N. Voitov, E.V. Boloev, L.V. Semenova, Izv. RAS Energetika 1, 25 (2018)

9. I.I. Golub, O.N. Voitov, E.V. Boloev, L.V. On Methodical problems of reliability research of large energy systems 67 , 141 (2016)

10. E. Mainika, Algorithms for optimization on networks and graphs (Mir, Moskow, 1981)

11. S.K. Gursky, Algorithmization of condition control problems for bulk power systems (Nauka i tekhnika, Minsk, 1977) 
12. O.N. Voitov, System studies in energy. Retrospective of scientific schools at SEI-ISEM, 324 (Nauka, Novosibirsk, 2010)

13. M.E Baran, F.F. Wu, IEEE Trans. Power Del. 4, 1401 (1989) 